\title{
Optical positions of radio stars. I.
}

\author{
$\mathrm{Hu} \mathrm{Hui}^{1}$ and Li Dongming ${ }^{2}$ \\ ${ }^{1}$ Yunnan Observatory, Academia Sinica, Kunming 650011, P.R. China \\ ${ }^{2}$ Purple Mountain Observatory, Academia Sinica, Nanjing 210008, P.R. China
}

Received August 17; accepted October 16, 1995

\begin{abstract}
The optical positions of 31 radio stars obtained from the observations with the photoelectric astrolabe at Yunnan Observatory are presented in this paper. These are all the stars in the astrometric catalogue of radio stars observable by our astrolabe.
\end{abstract}

Key words: astrometry — radio continuum: stars

\section{Introduction}

After being automated and equipped with a photon counter, the photoelectric astrolabe of Yunnan Observatory can automatically be operated to observe stars and the stars as faint as those of magnitude 11.0. The objects which can be observed with the instrument have been selected into the program from the astrometric catalogue of radio stars (Walter et al. 1990) in order to contribute to the link of the optical reference frame to the VLBI reference frame based on extragalactic objects. During one year of observations the optical positions of 31 radio stars are obtained from two transits each. The internal mean errors of the right ascensions and declinations are \pm 0.0037 and $\pm 00^{\prime \prime} 065$, respectively.

\section{Observations and reductions}

The observational and reduction procedure is the same one described in Hu Hui et al. (1994). Similarly, the same method described in the paper is adopted to calculate the differences YPA-CAT (where CAT stands for the positions in FK5 or CMC, and CMC stands for Carlsberg Meridian Catalogue), and the differences YPA-RSS (where RSS stands for the radio positions given in the astrometric catalogue of radio stars). The results indicate that the YPA's external accuracy is very good.

\section{Explanation of Table 1}

The resulting optical positions for the 31 radio stars are presented in Table 1.

Column 1: the number in the astrometric catalogue of radio stars.

Send offprint requests to: $\mathrm{Hu}$ Hui
Column 2: the number of FK5 or CMC, where the one that is larger than 1000 is that of the CMC.

Column 3: observed mean visual magnitude.

Columns 4 and 6: right ascension and declination for equator and equinox J2000.0 and epoch of observation.

Columns 5 and 7: mean errors of right ascension and declination.

Columns 8 and 9: the number of the observed transits in east and west, respectively.

Column 10: mean epoch of observations minus 1900.00.

Column 11: the root-mean-square error.

Columns 12 and 13: differences YPA-CAT in right ascension and declination, respectively. Definition of Da: $\mathrm{Da}=[\alpha(\mathrm{YPA})-\alpha(\mathrm{CAT})] \cos ^{\delta}$. The units of Da and $\mathrm{Db}$ are $0^{\prime \prime} 01$.

Columns 14 and 15: the differences YPA-RSS in right ascension and declination, respectively. The units of $\mathrm{Da}$ and $\mathrm{Db}$ are $0{ }^{\prime \prime} 01$.

Acknowledgements. The authors heartily thank the referee for valuable comments. The authors wish to express their gratitude to Dr. Wang Rui, engineer at Yunnan Observatory, for his participation in the observation together with the authors. This work is supported by National Natural Science Foundation of China (No. 19233010) and Chinese Astronomic Committee Foundation.

\section{References}

Carlsberg Meridian Catalogue La Palma No. 4, 1989; No. 5, 1990; No. 6, 1992

Hu Hui, Li Dongming, Wang Rui, Chen Cuixian, 1994, A\&AS 106, 103

Walter H.G., Hering R., de Vegt Ch., 1990, A\&AS 86, 357 
Table 1. 31 radio star positions. (Mean Julian epoch of observations; equator and equinox J2000)

\begin{tabular}{|c|c|c|c|c|c|c|c|c|c|c|c|c|c|c|}
\hline \multirow[b]{2}{*}{ No. } & \multirow[b]{2}{*}{$\mathrm{I}: \mathrm{K} 5 / \mathrm{CMC}$} & \multirow[b]{2}{*}{ Mg. } & \multirow[b]{2}{*}{$a$} & \multirow[b]{2}{*}{$m_{a}$} & \multirow[b]{2}{*}{$\delta$} & \multirow[b]{2}{*}{$\mathrm{m}_{5}$} & \multicolumn{4}{|c|}{ Epoch } & \multicolumn{2}{|c|}{ YPA-CAT } & \multicolumn{2}{|c|}{ YPA-RSS } \\
\hline & & & & & & & $N_{\mathrm{c}}$ & $N_{\mathrm{w}}$ & -1900 & $\sigma$ & $\mathrm{Da}$ & Db & $\mathrm{Da}$ & $\mathrm{Db}$ \\
\hline & & & h m $\mathrm{m}$ & 0 & $\cdots n$ & " & & & & $\pi$ & & $(\tilde{0.0 \mathrm{I})})$ & & \\
\hline 1025 & 107 & 2,49 & 30216778 & 0.002 & 40523.50 & 0.02 & 33 & 33 & 93.90 & 0.02 & 1 & 9 & & \\
\hline 1026 & 111 & 2.11 & $308 \quad 10.136$ & 0.001 & 405720.38 & 0.04 & 37 & 36 & 93.88 & 0.02 & 8 & -6 & 9 & 7 \\
\hline 2113 & 206 & 2.19 & 53200.398 & 0.006 & 01756.87 & 0.05 & 26 & 23 & 93.79 & 0.06 & 3 & 0 & & \\
\hline 1143 & 502 & 4.93 & 133447.759 & 0.003 & & & 21 & 36 & 93.31 & 0.03 & -1 & & 1 & \\
\hline 1265 & 890 & 3.80 & 233733.757 & 0.003 & 462731.98 & 0.03 & 28 & 34 & 93.77 & 0.03 & 1 & -5 & & \\
\hline 1010 & 200882 & 5.47 & 11636.340 & 0.004 & -23000.85 & 0.02 & 22 & 21 & 93.83 & 0.03 & 2 & 12 & & \\
\hline 1014 & 200944 & 6.13 & 12535.663 & 0.001 & & & 39 & 39 & 93.87 & 0.02 & -11 & & & \\
\hline 1028 & 202151 & 6.56 & 32635.364 & 0.001 & & & 37 & 26 & 93.89 & 0.02 & -7 & & -11 & \\
\hline 1036 & 202534 & 4.75 & 41814.583 & 0.005 & 501744.15 & 0.04 & 25 & 23 & 93.90 & 0.04 & 7 & -3 & -6 & -5 \\
\hline 1061 & 203137 & 7.83 & 54102.300 & 0.004 & -24300.80 & 0.03 & 34 & 28 & 93.91 & 0.04 & -10 & 3 & & \\
\hline 2658 & 403092 & 6.94 & 54126.771 & 0.003 & 34640.74 & 0.05 & 28 & 21 & 93.59 & 0.05 & 2 & -9 & & \\
\hline 2129 & 203320 & 9.25 & 60353.644 & 0.005 & & & 23 & 30 & 93.81 & 0,08 & 1 & & & \\
\hline 2166 & 305351 & 8.82 & 72724.164 & 0.005 & 153934.98 & 0.16 & 29 & 21 & 93.59 & 0.09 & 5 & 18 & & \\
\hline 1084 & 203994 & 4.36 & 74318.697 & 0.001 & & & 23 & 35 & 93.79 & 0.02 & -1 & & & \\
\hline 2183 & 504954 & 9.87 & 83908.542 & 0.006 & & & 25 & 21 & 93.61 & 0.10 & 0 & & & \\
\hline 2706 & 504987 & 8.66 & 84356.153 & 0.003 & & & 28 & 21 & 93.62 & 0.07 & 16 & & & \\
\hline 1118 & 308465 & 10.62 & 114046.367 & 0.011 & 515953.44 & 0.07 & 22 & 21 & 93.28 & 0.07 & 5 & 23 & & \\
\hline 1137 & 205167 & 8.32 & 131036.931 & 0.004 & & & 21 & 21 & 93.23 & 0.06 & -9 & & -11 & \\
\hline 1140 & 408965 & 8.25 & 133046.836 & 0.004 & & & 21 & 21 & 93.23 & 0.08 & 10 & & 9 & \\
\hline 1147 & 205365 & 6.81 & 135609.562 & 0.002 & & & 22 & 21 & 93.23 & 0.04 & -6 & & & \\
\hline 2298 & 311678 & 10.63 & 153915.242 & 0.004 & & & 27 & 30 & 93.35 & 0.06 & -8 & & & \\
\hline 2340 & 510310 & 8.32 & 171025.588 & 0.005 & 485756.43 & 0.04 & 21 & 31 & 93.30 & 0.04 & -12 & -12 & & \\
\hline 1189 & 206848 & 7.28 & 175806.999 & 0.005 & 150821.46 & 0.17 & 21 & 28 & 93.36 & 0.08 & -2 & 2 & 10 & 8 \\
\hline 2356 & 412354 & 9.74 & 175838.517 & 0.004 & & & 21 & 21 & 93.36 & 0.08 & -7 & & & \\
\hline 1207 & 207399 & 5.96 & 190825.858 & 0.007 & 522533.03 & 0.04 & 28 & 21 & 93.40 & 0.04 & -4 & -21 & & \\
\hline 2442 & 208226 & 8.10 & 201932.443 & 0.003 & 384353.86 & 0.11 & 22 & 30 & 93.55 & 0.05 & 17 & -21 & & \\
\hline 1225 & 413912 & 6.77 & 202027.983 & 0.003 & $4351 \quad 6.32$ & 0.06 & 21 & 26 & 93.57 & 0.03 & 0 & -9 & -3 & I1 \\
\hline 1227 & 316186 & 9.24 & 203222.431 & 0.005 & 411819.29 & 0.14 & 22 & 21 & 93.56 & 0.08 & -5 & 12 & 6 & 31 \\
\hline 2460 & $20883 !$ & 7.51 & 210225.870 & 0.002 & & & 26 & 32 & 93.62 & 0.03 & 7 & & & \\
\hline$\$ 251$ & 317881 & 6.39 & 223006.519 & 0.003 & 492123.40 & 0.03 & 28 & 25 & 93.74 & 0.03 & -5 & -6 & & \\
\hline \multirow[t]{5}{*}{1271} & 210622 & 5.89 & 234940.963 & 0,001 & & & 30 & 33 & 93.78 & 0.02 & 1 & & & \\
\hline & & & & & & & & & & & & \multicolumn{3}{|c|}{$(0.001)$} \\
\hline & Mcan & & & & & & & & & & -1 & -8 & 4 & 104 \\
\hline & Standard & etror of & nean & & & & & & & & 13 & 30 & 28 & 58 \\
\hline & Standard & deviation & & & & & & & & & 73 & 125 & 85 & 130 \\
\hline
\end{tabular}

